\title{
Introduction: Individual Concepts in Language and Thought
}

\author{
Tadeusz Ciecierski ${ }^{1} \cdot$ Paweł Grabarczyk ${ }^{2}$
}

Published online: 11 April 2019

(c) Springer Nature B.V. 2019

Individual concepts are concepts of individuals of a particular, arbitrary kind: particular persons, buildings, cities, particles, planets, numbers, sets, functions, qualities, events, processes or anything to which we can attribute properties. That is, individual concepts are devices for speaking and thinking about particular entities no matter what the ontological status of the entity is. What makes a concept individual rather than of individuals? Answers to this questions may follow Leibniz's observation that 'some concepts do completely determine an actual or possible individual, distinguishing it in one respect or another from every other possible individual' (Mates 1989: p 62). ${ }^{1}$ This initial and basic characterisation, however, is oblivious to several important philosophical distinctions and controversies; it suffers also from ambiguities connected with the word 'concept'.

The first important distinction that has to be taken into the account here is the one between the psychological and non-psychological meaning of 'concept' (in fact, we should rather write 'meanings' as there are many psychological and non-psychological uses of the word in the philosophical, psychological and linguistic literature). Although some authors, despite Frege's warning, occasionally equivocate between the two meanings, ${ }^{2}$ while others attempt to reduce concepts in the second sense to concepts in the first sense, ${ }^{3}$ the difference should be clear and uncontroversial. Concepts in the psychological sense or, as some prefer, mental representations, are constituents of mental states and (possibly) elements of the body of knowledge actually employed in higher cognitive processes. ${ }^{4}$ Concepts in the non-psychological sense, on the other hand, are entities accessible intersubjectively that may be used to classify thoughts and expressions

Tadeusz Ciecierski

taci@uw.edu.pl

Paweł Grabarczyk

pagrab@gmail.com

1 Institute of Philosophy, University of Warsaw, Warsaw, Poland

2 IT University of Copenhagen, Copenhagen, Denmark as content-identical, content-similar, and so forth. As such, they may be the results or products of acts of theorising, or the inputs and outputs of procedures such as explication.

The phrase 'individual concept' - in its contemporary (non-Leibnizian) sense-was coined by Rudolf Carnap ${ }^{5}$ and used solely in the non-psychological and linguistic sense. For Carnap, individual concepts were intensions of individual expressions: constants, descriptions and individual variables. Carnap applied this category (like all the others that were relevant for his method of extension and intension) solely to artificial languages he called semantic systems. One may assume,

\footnotetext{
1 Mates adds to this: 'such concepts are called by Leibniz "complete individual concepts". Thus, a complete individual concept contains every attribute of every individual that can fall under it; it resolves every question that could be raised about such an individual-that is, it determines exactly one possible individual' (Mates 1989, pp. 62-63). Here Mates takes the uniqueness of some attribute of $x$ as tantamount to a total collection of every attribute $x$ has. However, the properties distinguishing $\mathrm{x}$ from every other possible individuals does not have to be total and complete. To distinguish 'complete individual concepts' and regular 'individual concepts' we might use the terminology coined by Edward Zalta and speak (in the former case) about the blueprints of individuals (cf. Zalta 1983, p. 35).

${ }^{2}$ Susan Carey writes, for instance, that concepts are 'units of thought, the constituents of beliefs and theories' (Carey 2011, p. 5). It is, however, clear that concepts qua constituents of beliefs are not concepts qua constituents of theories conceived as the results or products of theory construction.

3 Cf. Margolis and Laurence (2007).

${ }^{4}$ It is rather likely that this category is far from being homogenous (cf. Machery 2010).

${ }^{5}$ Carnap (1947). 'We have earlier found entities which seemed suitable as intensions of designators of other types; for sentences, propositions; for predicators, properties or relations; for functors, functions. Thus, in these cases, the intensions are those entities which are sometimes regarded as the meanings of the expressions in question; and, in the case of predicators and functors, the intensions are concepts of certain types. Now it seems to me a natural procedure, in the case of individual expressions, likewise to speak of concepts, but of concepts of a particular type, namely, the individual type. Although it is not altogether customary to speak here of concepts in this sense, still it does not seem to deviate too much from ordinary usage. I propose to use the term "individual concept" for this type of concept.' (pp 40-41).
} 
however, that this category can be applied to languages more broadly and to individual expressions of natural languages, such as proper names, descriptions and indexical singular terms (like deictically used personal pronouns). Given this we may consider the following claim or hypothesis:

(Carnap Thesis) Every individual expression (of a possible language $\mathrm{L}$ ) has an intension. The intension of an individual expression is an individual concept.

But what exactly are individual concepts in this sense? Carnap's initial explanation appealed to notions such as L-equivalence and the linguistic counterparts of possible worlds called state-descriptions. Contemporary practitioners of intensional logic will express Carnap's idea by saying that individual concepts are functions from possible worlds to individuals (or more broadly: from circumstances of evaluation to individuals). Thus interpreted, individual concepts were used by authors like Scott and Montague in their inquiries into intensional semantics. ${ }^{6}$

Depending on what particular individual expression is at stake, such functions may be constant or variable. If they are constant, we may say that the corresponding expressions are rigid. If we think that the functions are constant and total, we may say that the corresponding expressions are Kaplan rigid. On the other hand, if they are constant but partial, we may say that they are Kripke rigid. ${ }^{7}$ Neither Kaplan rigidity nor Kripke rigidity should be confused with direct reference. Neither implies that the reference of an expression is direct in the sense of not requiring identifying properties or modes of presentations to mediate between individual expressions and their referents. This can best be seen in the case of rigid definite descriptions (e.g. 'the president of the USA in 1918 in LEIBNIZ' where 'LEIBNIZ' is the name of the world we live in) which are not directly referential, but which may be Kaplan rigid if a certain semantic treatment of descriptions is assumed-that is, the one that takes descriptions to be referential terms while assuming that the corresponding individual concepts have no value in the worlds where the individual in question (Woodrow Wilson) does not exist.

There is definitely an interpretation of the notion of the individual concept (in terms of individuating qualities or descriptive modes of presentation) and the interpretation of the notion of direct reference that might result in the refutation of the Carnap thesis if some individual expressions are directly referential (and it is very likely that some, like proper names or indexicals, are). This is what Kaplan had in mind when he wrote that if there are directly referential terms, then

\footnotetext{
${ }^{6}$ For a relatively recent application of the idea, see Aloni (2005), which discusses various frameworks that are philosophically rather controversial as being committed to the idea of contingent identity.

${ }^{7}$ Cf. Steinman (1985).
}

the 'proposition expressed by a sentence containing such a term would involve individuals directly rather than by way of the individual concepts or manners of presentation I had been taught to expect' (Kaplan 1989: p 483).

However, other interpretations of the notion of the individual concept are possible and it is far from being certain that the Carnap thesis is inconsistent with the observation that some individual expressions are directly referential. Carnap nowhere explained the idea of the individual concept in terms of individuating qualities or descriptive modes of presentation, and this clearly enables the interpretation that it as nothing more than a description of specific modal behaviours of expressions of certain categories. In this spirit, Abbott (2011) has recently suggested that constant individual concepts might be very useful in the analysis of empty proper names and specific uses of indefinite descriptions, while variable individual concepts might be useful in the analysis of nonspecific uses of indefinite descriptions.

If the propositional content (of a certain sentence or utterance) contains an individual as a constituent (this usually means that the sentence or utterance in question contains a directly referential term that refers to that individual) numerous authors will call it a singular proposition. However, if we agree that Carnap's notion of the individual concept is not inconsistent with direct reference, we may also think of singular propositions as ones that contain rigid individual concepts as constituents.

All in all, the basic problems connected with the Carnap thesis and the linguistic interpretation of the notion of individual concept are: (i) possible explications of the latter, in terms of manners of presentation, descriptive and nondescriptive de re senses (cf. McDowell 1984), kinds of constructions (cf. Tichy 1986) or encoding and exemplification (Zalta 1983); (ii) the issue of its applicability to particular classes of expressions or uses of expressions (e.g. proper names, indexicals, definite and indefinite descriptions, fictional names and de se and non-de se attitude reports); and (iii) the problem of the modal behaviour of individual concepts that correspond to particular expressions or uses of expressions - that is, is this individual concept (or kind of individual concept) Kaplan rigid, Kripke rigid or non-rigid?

The second important distinction regarding the idea of the individual concept is that one must distinguish between theories of concepts that attempt to treat concepts in the non-psychological sense as the basis for a joint classification of thoughts and expressions and an alternative theories that enable disjointed classifications of the two. The former can be viewed as friendly towards the so-called homogeneity hypotheses ${ }^{8}$ according to which there exists a single kind

\footnotetext{
${ }^{8}$ See Weber (2012). This kind of stance is implicitly taken by philosophers who argue for object-dependent thoughts by appealing to direct reference (cf. Adams et al. 1999, p. 47).
} 
of entity that is (or plays the role of) both mental and linguistic content. Homogeneity enables direct application of the categories worked up in the theories of language (like intension, singular proposition or general proposition) to intentional states or attitudes. The opposite view, for heterogeneity, should work up its own category of singularity as a property of intentional states. One possibility (not reserved for proponents of heterogeneity) is to use the notions introduced in the debate concerning mental representations (i.e. concepts in the psychological sense).

In the domain of mental representations, the close counterpart of the notion of singular proposition is the concept of the object-dependent thought. This concept was introduced by Gareth Evans, who held that:

there are thoughts we have about particular objects which we simply could not have if those objects did not exist. For example, an internal state of a subject can be ascribed the content that this table is round only if there is a particular object it is about, on which it is causally dependent. There is no neutral or existenceindependent specification of this content to which we can retreat if the subject is hallucinating. If there is no object (whether or not the subject believes there is), there is no content—no thought. (Evans 1985, p. 402) ${ }^{9}$

As Evans suggested, object-dependent thought is usually explained in terms of two slogans: Change the object, change the thought and No object, no thought (cf. Corazza 2004). The debate around object-dependent thoughts mostly revolves around the question of whether psychological generalisations or laws involving object-dependent thoughts are possible (cf. Noonan 1991; Adams et al. 1999; Corazza 2004), the related issue of the connection of the view to internalism in the philosophy of mind (cf. Adams et al. 1993) and the (also related) question about the role of object-dependent thoughts in causing actions (and the role of the concept in theories that attempt to explain actions). It is rather a popular view that one should not believe in the existence of object-dependent thoughts unless they manifest themselves in actions and unless the concept of objectdependent thought plays a role in action description and action explanation.

\footnotetext{
${ }^{9}$ Elsewhere, Evans explicates the notion in terms of informational connection: "Consequently, demonstrative thoughts about objects, like "here"-thoughts, are Russellian. If there is no one object with which the subject is in fact in informational "contact"-if he is hallucinating, or if several different objects succeed each other without his noticing - then he has no Idea-of-a-particular object, and hence no thought. His demonstrative thought about a particular object relies upon the fact of an informational connection of a certain kind, not upon the thought or idea of that connection; and hence it is unconstruable, if there is no object with which he is thus connected' (1982, p. 173).
}

Even if object-dependent thoughts exist, it is neither likely that all singular mental representations are object dependent, nor that the latter category is homogenous. In particular, philosophers are very interested in the class of indexical attitudes (and their representations) such as beliefs about 'where one is, when it is, and who one is' (Perry 1979: p 5). Beliefs of the last kind are known in the literature as de se beliefs. Authors such as Feit and Capone (2013) distinguish here 'the psychological problem of de $s e$ '. The challenge here is to 'identify the content of a given de se belief' and to distinguish it from the content of beliefs that are not de se (Feit and Capone 2013). They also single out the linguistic problem of de se (what is the semantic value of 'I', 's/he herself' or self-oriented long-distance reflexives in belief clauses) and the generality problem identified by Geach ('How can distinct people share the same de se belief?'). The linguistic problem of the de se belongs properly to the class of issues connected with nonpsychological understanding of individual concepts, while the psychological problem of de se belongs to the class of issues connected with psychological understanding of individual concepts. However, because the authors interested in the latter problem assume homogeneity, they may consider the issue to also be closely connected with the semantics of de se reports.

Because the main distinction between concepts in the psychological and non-psychological sense applies to concepts in general, it might be directly employed to particular kinds of concept - concepts of individuals of an arbitrary ontological category included. The distinctions sketched above give us the following rough and initial scheme:

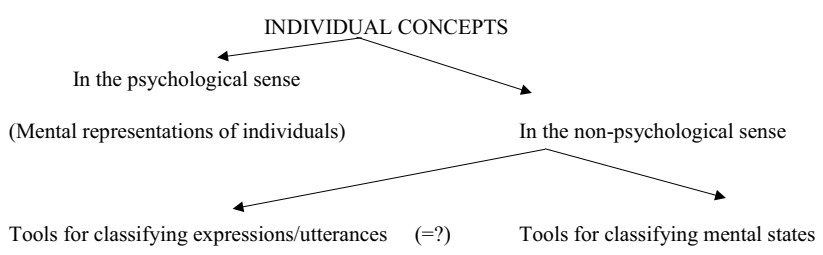

The papers presented in this issue consider the notion of individual concepts in all three distinguished senses and address the majority of problems described above.

This issue starts with two papers centring on the debate between descriptivism and anti-descriptivism. In 'Names, descriptions and causal descriptions. Is the magic gone?', Marti discusses critically the idea of causal descriptivism (cf. Kroon 1987; Lewis 1997) - the view arising partially in reaction to the observation that anti-descriptivism regarding proper names does not offer an explanation of the referential connection between a name and its bearer. The main idea behind causal descriptivism is building information about the causal communicative chain that connects the referent of 
$\mathrm{N}$ with a particular utterance of $\mathrm{N}$ into the reference-fixing description. Marti argues that causal descriptivism-contrary to its initial promises-does not provide the required explanation of the referential link, so it offers no better theory in that respect than the new theory of reference..$^{10}$ The paper also contains remarks about the anti-descriptivism of Ruth Barcan Marcus and about the general explanatory demands concerning the nature of naming relation.

In 'Descriptivism without quotation' Franken presents the idea of inter-nominal descriptivism. This view arises in opposition to quotational versions of descriptivismthat is, views that assume that the descriptive contents of names always involve their quotations (the author interprets causal descriptivism as a theory committed to this view). Franken sketches a descriptivist theory that is free from this assumption. First, he proposes that all particular proper names of natural languages (or rather: their uses in contexts) are in fact particular realisations of a single abstract proper name (referred to as 'NAME'). He proposes thinking of particular names (or their uses) as analogous to particular uses of indexicals and about the abstract proper name as analogous to an indexical type. Uses of names, according to the Franken, have no meaning that can be properly attributed only to the NAME and which is analogous to Kaplanian character. Following Kapitan (2016), he distinguishes between executive and interpretative modes of presentations for expressions - that is, the one that is executed by the speaker and the other (token, reflexive one) that is executed by the person who interprets the utterance. The character of a NAME is, therefore, a pair of the two kinds of modes of presentation that directly refer to particular tokens of a NAME. Franken defines both modes of presentation for NAME and closes the paper with a discussion of the problems inter-nominal descriptivism must face.

The next paper in the volume, 'Proper Names, Rigidity, and Empirical Studies on Judgments of Identity Across Transformations', by Dranseika et al., is a critical excursion into meta-psychology that analyses the popular mode of inference used in studies of folk judgements of objects' identity across transformations. The authors are especially interested in the studies of folk opinions regarding scenarios without identity assumptions- that is, the ones where the studied subjects are asked whether the object's identity is preserved when central transformations take place. The strategy involves three steps: (i) the descriptions of the transformation scenario, (ii) the investigation checking whether the studied subjects treat pre-transformational and post-transformational

\footnotetext{
10 As Marti stresses, however, it is a mistake to attribute to all new reference theorists a single view consisting in commitment to a particular kind of truth-conditional contribution of singular terms.
}

entities as identical and (iii) drawing conclusions on the basis of answers to (ii) about the relevance of certain criteria as folk criteria of identity across transformations. The authors are especially interested in the strategy of taking uses of proper names of objects as criteria for assessing the results obtained at stage (ii) of the study. They defend the view that the assumption of the rigidity of names-used in the justification of the strategy - cannot help in assuring that the conclusions drawn from the experimental studies are correct. First, the use of the assumption does not preclude the possibility that a proper name is used differently by the studied subject. Second, the authors claim that the use of the rigidity assumption in the experimental study is always committed to the empirical (and empirically controversial) claim that a particular expression is used rigidly. Third, they argue that the use of the assumption to draw conclusions about the attitudes of the subjects involves 'a significant and possibly irreducible amount of circularity and indeterminacy'. The problem discussed in the paper has interestin relationship to the problem of the substitution of co-referential terms in non-intensional contexts (cf. Poller 2016).

In 'Truth Without Reference: The Use of Fictional Names', de Ponte et al. address the issue of reconciling two intuitive observations: that fictional names have no referents and that statements that contain them in many cases seem to have truth value. The authors are especially interested with parafictional statements - that is, statements about fictional states of affairs made outside fiction by actual persons (like 'Holmes and Watson were friends.' or 'Poirot was Belgian.' as uttered in regular conversations). The analysis provided in the paper is inspired by critical referentialism (Perry 2012) and is based on two concepts: the concept of network and the concept of notion. First, the authors distinguish three (interdependent) components of the truth conditions of all statements containing singular terms: the existential condition, the reference condition and the satisfaction condition. The first consists in the statement that a particular object exists, the second (dependent on the first) is the statement that the use of a singular term refers to that object and the third one is the statement that the object in question has the property attributed to it in the statement. Second, the authors elaborate the reference condition by building into it the concept of a network of conditional co-reference (coco-reference), namely that a particular use of a name is normally associated with the intention of using it in accordance (co-referring) with some of its earlier uses. Normally the speaker presupposes that the later use refers if the earlier use refers (hence conditional co-reference). This enables the speaker to create (or enter) the entire network of conditional co-reference and (normally) refer to the origin of that network, that is refer to the object that is at the beginning of the network. So, given a name $n$, an utterance $u$ (containing $n$ ) we might formulate the reference condition as stating that there exists 
a coco-reference network $\mathrm{N}_{\mathrm{n}}$ (containing $n$ ) exploited in the utterance of $u$ that has a particular object (mentioned explicitly in the existence condition) as its origin. Every network can be associated with a notion-that is, a cluster of information (and misinformation) about an individual that is the origin of the network; more generally, notions are clusters of information and misinformation concerning individuals we think and talk about. Every use of a proper name therefore has a corresponding notion-network structure. However, fictional uses of names (occurring in parafictional statements) are specific since their networks end in blocks. They are also generally not purported to refer to their origin. They therefore differ from other kinds of empty uses of names that might be purported to refer to their supposed origins ('Vulcan' is historical example of this sort, but regular fictional names might also be used in this non-fictional sense by persons who take them to be non-fictional). The authors therefore suggest that, properly understood, the parafictional statements are in fact statements about notions and networks (and not about non-existent referents of fictional names). This requires a change in the (elaborated) existential conditions, reference conditions and satisfaction conditions of statements. For instance, the conditions for the statement (A) 'Poirot was Belgian' become:

(Existential Condition) The notion $\mathbf{n}_{\text {Poirot }}$ exists.

(Reference Condition) There exists an originless network $\mathbf{N}_{\text {Poirot }}$ such that the use of 'Poirot' in 'Poirot was Belgian' exploits $\mathbf{N}_{\text {Poirot }}$ and $\mathbf{N}_{\text {Poirot }}$ ends in the notion $\mathbf{n}_{\text {Poirot. }}$.

(Satisfaction Condition) the notion $\mathbf{n}_{\text {Poirot }}$ includes the idea of being a Belgian.

While the conditions for (B) 'Poirot was a ballet dancer' are:

(Existential Condition*) The notion $\mathbf{n}_{\text {Poirot }}$ exists.

(Reference Condition*) There exists an originless network $\mathbf{N}_{\text {Poirot }}$ such that the use of 'Poirot' in 'Poirot was a ballet dancer' exploits $\mathbf{N}_{\text {Poirot }}$ and $\mathbf{N}_{\text {Poirot }}$ ends in the notion $\mathbf{n}_{\text {Poirot }}$.

(Satisfaction Condition*) the notion $\mathbf{n}_{\text {Poirot }}$ includes the idea of being a ballet dancer.

Since the corresponding satisfaction conditions respectively are and are not met, we might treat the parafictional statement (A) as true and the parafictional statement (B) as false. The authors supplement this approach with additional analysis of uses of (ordinary) empty names and negative existential statements, exploiting the view that such statements do not have existential and satisfaction conditions.

The problem of fiction is also the topic of the next paper. In 'How to Pick Out a Dragon: Fiction and the Selection Problem', Haraldsen discusses the relationship between possibilism and the so-called selection problem. Possibilism (about fictional objects) claims that fictional entities are merely possible objects (existing in some possible worlds but not in the actual one). Haraldsen defends the view that possibilism cannot be questioned on the basis of purely semantic considerations and that, if it is incorrect, this is so rather due to metaphysical (and non-semantic) reasons. This conflicts with the opinions of authors such as Thomasson (1999), Sainsbury (2010) and Kripke (2011). According to these authors, the main challenge to possibilism is the selection problem: the issue of reconciling three claims presupposed by possibilists. The first (uniqueness) is that, for a particular fictional object $f$ (Sherlock Holmes, Hercule Poirot, the city of Utopia, etc.) there is at most one $f$ (at most one Sherlock Holmes, Hercule Poirot, Utopia etc.). The second (multiplicity) is that there are many $f$-candidates in distinct possible worlds-that is, objects that satisfy the entire descriptive content associated with $f$ (there are many Sherlock Holmes-candidates, Hercule Poirot-candidates or Utopia-candidates, etc.). The third (arbitrariness) is that we have no criteria for selecting one of the $f$-candidates (and not others) as the entity being $f$. Haraldsen defends the view that we may successfully fix the reference of a particular fictional name in the process of arbitrary selection (by stipulation) and that story-telling might be conceived of as narrowing down the set of possible worlds in which the selected individual exists by attributing more and more properties to it. Haraldsen also defends the view that the sketched possibilist account is consistent with the causal theory of reference.

The next paper in the issue, by Moldovan, concerns the case of non-denoting descriptions. According to the referential treatment of definite descriptions (originating with Frege and Strawson), statements like (M1) 'The present king of France is sitting in that chair' and (M2) 'The present king of France is not sitting in that chair' lack truth values. At the very same time they are, respectively, intuitively false and true. Referentialists are, therefore, expected to have a pragmatic explanation of the intuition in question. Moldovan criticises existing pragmatic explanations of this sort of intuition (following von Fintel 2004; Yablo 2006) while also offering his own explanation. The pragmatic approach of von Fintel assumes the strategy of epistemic revision and the rejection or acceptance of utterances based on revision. The idea is that one has a disposition to judge utterances like M1 to be false due to reasons independent of the fact that the description is non-denoting. Roughly speaking, (M1) is judged to be false because even if the present king of France did exist, he is not actually sitting in that chair. Moldovan provides counterexamples to this explanation: he argues that the strategy has problems with correct predictions in cases of certain utterances that are intuitively false (one of his examples is: 'The natural satellite of the Moon is a component of the Solar System') while having a more general problem with explaining the intuitions of truthfulness in cases like (M2). He also criticises the theoretical motivation behind the 
approach that is committed to either interpreting the epistemic revision in terms of accommodation or interpreting it in terms of belief revision. He argues that neither of the two interpretations is tenable. He argues that Yablo's alternative analysis also gives erroneous predictions and cannot be treated as theoretically superior to von Fintel's. In presenting his own explanation, Moldovan agrees in spirit with von Fintel's diagnosis that utterances like M1 are judged to be false due to reasons independent of the fact that the description is non-denoting. However, he proposes that these reasons are the result of taking certain (contextually relevant) entailments of utterances to be the basis of modus tollens reasoning that results in the rejection of the original sentence (and in attributing falsehood to that sentence). Moldovan's proposal enables the analysis of problematic cases.

Is content (or at least some of its aspects) transparent to language users? The answer to this question bears an interesting relation to the discussion regarding the content of sentences and utterances that contain empty singular terms. As pointed out by Hodgson, the notion of content transparency is ambiguous, because it may actually refer to three separate things. According to the strongest interpretation, users should be able to detect differences in the contents of expressions. According to the weaker version, the only ability users are expected to have is the ability to detect differences in the structure of the content of expressions. According to the weakest version of the transparency thesis, it is only the structural type (e.g. the difference between singularity and generality) of content that has to be detectable by competent users of the language. The first option (transparency of content) is, as Hodgson points out, highly implausible. Hodgson also refutes the weaker senses of transparency. He argues that differences in the structure of content may not be detectable due to the existence of unarticulated constituents. $\mathrm{He}$ also formulates a challenging dilemma for the proponents of structural transparency: they have to provide justification for their claim free of petitio principii. He closes the paper with a criticism of the positive arguments for transparency.

The next paper in the collection, by Sean Crawford, concerns the content of thoughts and utterances containing perceptual demonstratives (PD) and the applicability of the notion of object dependency to such thoughts and utterances. Crawford proposes an in-between position between theories committed to the object dependency of PD and the ones committed to the object independency of PD; he argues for the property-dependent theory of perceptually demonstrative thoughts and utterances-that is, the view that thinks of the content of perceptual demonstrative thoughts as constituted by the observable properties of objects (not as constituted by their essences or identities). Once we combine this idea with the view according to which hallucinations are not treated as bona fide perceptions, we can arrive at the following conclusion: contents of hallucinations are neither object nor property dependent, as hallucinations do not provide us either with objects or with properties that we can - they involve only the phenomenal side of the experience, not the actual perception of anything (in this respect the property-dependent theory is close to the No object, no thought motto of object-dependency theorists). The difference between Crawford's account and object-dependency theories becomes noticeable once we look at a different set of problematic cases: phenomenal duplicates. In some situations, the perceiver may have a phenomenally identical perception coming from a numerically different object. According to theories that posit object-dependent content, in such situations the content must be different. The solution suggested by Crawford is much more convincing, because the duplicate objects can be said to contain the same set of properties, so the content of the corresponding thought is the same.

Another solution that aims at an 'in-between' position can be found in the paper by Voltolini, who frames his proposal as an opposition the existing ideas found in Crane (2013). According to Crane, the tension between the idea that some expressions have their content fixed by objects to which they refer can be mitigated by the distinction between what the expressions refer to and what they purport to refer to. According to this idea, expressions that fail to refer can still retain their content, as they still function 'as if' they were referring to singular objects (as opposed to properties, sets or universalia). Content, therefore, can be said to be fixed more by how the referring mechanism is designed than by what it actually refers to. The phenomenal side is an important aspect that Crane adds to the mix here: the fact that these expressions 'feel' identical as expressions that successfully refer to singular objects plays an important role. Voltolini argues that these new additions to the notion of singular expressions are not necessary and that a similar solution can be provided by a simpler theory that does not demand a new notion of the singular thought. He proposes the differentiation between the notion of 'reaching' the target and 'constituting' the target. Using a helpful analogy to mereological sets, he points out that, just as we do not expect parts constituting the mereological whole to 'reach' it (whatever it would mean in this context), we do not have to expect the expression to reach the object that constitutes it. Whether this counter-proposition really does not presume a new notion of singular thought may be arguable, but it is definitely an interesting new take on the subject.

From the intuitive, non-critical standpoints it seems obvious that it should be possible for various agents to perform the same type of action. This common intuition is both reflected in our speech when we talk about different people doing 'the same thing', as well as in our folk-psychological practices of action explanation (as is explicitly assumed in Geach generality problem mentioned above). 
Yet, as is pointed out by Verdejo, the connection between actions and their intentional explanation involving de se beliefs makes this intuition-which Verdejo spells out in terms of public action requirement-somewhat dubious. The reason for it is that if de se attitudes are agent dependent and if actions depend on these attitudes then, one may argue, we are committed to action explanations that are agent specific. To solve this problem, Verdejo distinguishes three possible levels at which action-explanation can be conducted-the agent-independent, agent-bound and agent-specific levels. The difference between the second and third (the first is not useful in dealing with the problem, because it relates only to actions that are not tied to de se beliefs) is that, whereas agent-specific actions demand a particular user to be connected to an action-as such, they cannot be shared amongst many agents-the category of agent-bound actions do not presume anything specific about the agent. According to Verdejo, the proper locus of action explanation (involved especially in the well-known Perry cases of actions connected with de se attitudes) that is consistent with the public action requirement can be located at the agent-bound level. Taking this into account indicates the constraints both for proponents of essentially indexical action explanations (they have to account somehow for the shareability of actions) as well as for philosophers friendly towards the possibility of impersonal action explanations (they have to take into the account the insights from Perry cases).

In the last paper of this volume, Dobler addresses the problems posed by so-called 'Travis cases'. Travis cases show us that the phenomenon of context sensitivity extends far beyond the typical examples of personal pronouns and demonstratives and can be replicated even in some of the most 'innocent' circumstances of predication. An example of such a situation might be the answer to the question 'Is the grass in the garden green?' delivered by two people: a decorator who painted the grass so it looks green and the botanist who wishes to study the grass. The problem that this example poses is that the same expression seems to change its truth value depending on the speaker, although it does not seem to contain any singular expressions that are typically responsible for such shifts. Dobler presents a new take on this problem and suggests that what changes in these cases is not the truth condition of the sentence, but its acceptance conditions. The main idea behind this approach is that users accept or reject sentences or utterances in the context of some of the goals they have in mind. This causes users to selectively ignore some of the circumstances that, given different goals, would have made them accept the utterances. To return to our example: the botanist ignores the fact that the grass looks green, because it is irrelevant from the perspective of her goal-the botanical study of grass. The downside of this solution is that Dobler has to assume an error theory according to which the users reject sentences that they know to be true. Dobler argues, however, that this consequence is not particularly problematic. Although Dobler does not use this example, her solution seems to be corroborated by some of the expressions users invoke in Travis-like cases. For example, it would be quite natural for the botanist to say 'It is technically true that the grass is green, but it is not green'.

Acknowledgements We would like to thank all of the authors for the high quality of their contributions, the reviewers for their deep and accurate reviews and the Topoi team for all their help during the long editorial process. We would also like to mention that some of the papers in this collection have been presented at the first Context, Cognition and Communication Conference held at the University of Warsaw in June 2016.

\section{References}

Abbott B (2011) Support for individual concepts. Linguist Philos Invest 10:23-44

Adams F, Stecker R, Fuller G (1993) Thoughts without objects. Mind Lang 8:90-104

Adams F, Stecker R, Fuller G (1999) Object-dependent thoughts, perspectival thoughts and psychological generalization. Dialectica 53:47-59

Aloni M (2005) Individual concepts in modal predicate logic. J Philos Logic 34:1-64

Carey S (2011) Precis of the origin of concepts. Behav Brain Sci 34:113-124

Carnap R (1947) Meaning and necessity. University of Chicago Press, Chicago

Corazza E (2004) Reflecting the mind. Oxford University Press, Oxford

Crane T (2013) The objects of thought. Oxford University Press, Oxford

Evans G (1982) The varieties of reference. Oxford University Press, Oxford

Evans G (1985) Collected papers. Clarendon Press, Oxford

Feit N, Capone A (2013) The problem of de se attitudes. In: Feit N, Capone A (eds) Attitudes de se. Linguistics, epistemology, metaphysics. CSLI Publications, Stanford

Kapitan T (2016) Indexical duality: a Fregean theory. Rivista internazionale di Filosofia e Psicologia 7:303-320

Kaplan D (1989) Demonstratives. In: Almong J, Wettstein H, Perry J (eds) Themes from Kaplan. Oxford University Press, Oxford

Kripke S (2011) Philosophical troubles: collected papers, vol 1. Oxford University Press, Oxford

Kroon F (1987) Causal descriptivism. Australas J Philos 65:1-17

Lewis D (1997) Naming the colours. Australas J Philos 75:325-342

Machery E (2010) Doing without concepts. Oxford University Press, Oxford

Margolis E, Laurence S (2007) The ontology of concepts-abstract objects or mental representations? Nous 41:561-593

Mates B (1989) The philosophy of Leibniz. Oxford University Press, Oxford

McDowell J (1984) De re senses. Philos Q 34:283-294

Noonan H (1991) Object-dependent thoughts and psychological redundancy. Analysis 51:1-9

Perry J (1979) The problem of the essential indexical. Nous 13:3-21 
Perry J (2012) Reference and reflexivity. CSLI Publications, Stanford Poller O (2016) All the superheroes' names. Studia Semiotyczne 30(2):11-44

Sainsbury M (2010) Fiction and fictionalism. Routledge, London

Steinman R (1985) Kripke rigidity versus Kaplan rigidity. Mind 94:431-442

Thomasson A (1999) Fiction and metaphysics. Cambridge University Press, Cambridge

Tichy P (1986) Constructions. Philos Sci 53:514-534

von Fintel K (2004) Would you believe it? The king of France is back! (Presuppositions and truth-value intuitions). In: Reimer M, Bezuidenhout A (eds) Descriptions and beyond. Oxford University Press, Oxford
Weber C (2012) Eternalism and Propositional Multitasking: in defence of the Operator Argument. Synthese 189(1):199-219

Yablo S (2006) Non-catastrophic presupposition failure. In: Thomson JJ, Byrne A (eds) Content and modality: themes from the philosophy of Robert Stalnaker. Oxford University Press, Oxford

Zalta E (1983) Introduction to axiomatic metaphysics. D. Reidel Publishing Company, Dordrecht

Publisher's Note Springer Nature remains neutral with regard to jurisdictional claims in published maps and institutional affiliations. 